Ying F. and Tookey J. (2017). "Key Performance Indicator for Managing Construction Logistics

Performance." In: LC3 2017 Volume II - Proceedings of the 25th Annual Conference of the International

Group for Lean Construction (IGLC), Walsh, K., Sacks, R., Brilakis, I. (eds.), Heraklion, Greece, pp. 869-

876. DOI: https://doi.org/10.24928/2017/0013

\title{
KEY PERFORMANCE INDICATOR FOR MANAGING CONSTRUCTION LOGISTICS PERFORMANCE
}

\author{
Fei Ying ${ }^{1}$ and John Tookey ${ }^{2}$
}

\begin{abstract}
Construction logistics is an essential part of lean construction for both project management and cost aspects. The quantum of money that is embodied in the transportation of materials to site could be 39 to $58 \%$ of total logistics costs and between 4 to $10 \%$ of the product selling price for many firms (Coyle, Bardi et al. 2003). However, limited attention has been paid to this issue in the New Zealand construction industry. The purpose of this paper is to contribute to the knowledge about managing transportation costs by setting a Key Performance Indicator based on the number of vehicle movements to the construction site. A case study approach was adopted with on-site observations. Observations, including vehicle movements and material delivery patterns, were performed from the start of construction until "hand-over" to the building owner. Data analysis of vehicle movements suggested that construction transportation costs can be monitored and managed. The identified number of vehicle movements as a key performance indicator offers a significant step towards logistics performance management at the operational level in construction projects. It provides a basis for benchmarking that enables comparison, learning and improvement and thereby continuous enhancement of best practice.
\end{abstract}

Keywords: Lean construction, construction logistics, KPI, New Zealand.

\section{INTRODUCTION}

Lean construction has been adopted by the construction industry as a means of Supply Chain Management (Jorgensen and Emmitt 2009). The aim of lean construction is to work on continuous improvement, waste elimination, strong user focus, value of money, high quality management of projects and supply chains, and improved communications (OGC 2000). The construction industry is a project-based industry where each project is unique. This uniqueness has a direct influence over the implementation of lean construction. Furthermore, each project presents a logistical exercise customised to fit its operations. Since the industry does not elect where it conducts its productive activities, it therefore has to move where the work is. Construction logistics covering materials management and physical distribution is thus an essential part of lean construction. Considering that materials usually account for between 30 to over 50 percent of the cost of a building project (Fellows, Langford et al. 2002), transportation costs represent approximately 39 to 58 percent of the total logistics costs resulting in between 4 to 10 percent of the product selling price for many firms (Coyle, Bardi et al. 2003). The transportation costs of material hence represent a substantial percentage of the cost profile of the construction industry.

Lecturer, Department of Built Environment, Auckland University of Technology, Auckland, New Zealand, fei.ying@aut.ac.nz

2 Professor, Department of Built Environment, Auckland University of Technology, Auckland, New Zealand, john.tookey@aut.ac.nz 
Therefore, a small percentage cut in transportation costs could bring a sizable increase in profits.

Key performance indicators (KPIs) are widely used internationally to measure the performance of the construction industry (The KPI Working Group 2000). In New Zealand, a series of KPIs based on those developed in the United Kingdom (UK) have been measured over five years. Ten KPIs endorsed by the government address both project and company performance. These KPIs focus on construction cost, construction time, predictability of cost and time, defects, client satisfaction, safety, profitability and productivity (Constructing Excellence (NZ) Ltd 2008). Although the government intends to endorse a broad set of practical indicators, there is no KPI to address the performance of logistics costs and efficiency. Considering the sizable expenditure involved in transport related activities, it is therefore paradoxical that there is no appropriate KPI to measure logistics performance in the construction industry.

The aim of this paper is to propose that vehicle movements to the site can be used as KPI in monitoring and improving logistics performance. The focus is to measure transportation costs at both project and company level. To demonstrate the application of a proposed KPI, a case study of a commercial project in major New Zealand city is assessed. The findings of the project offer insights into the implementation of such an indicator to measure material transportation costs and monitor logistics performance.

\section{Performance Measurement in CONSTRUCtion Logistics}

Logistics is the umbrella term covering materials management and physical distribution (Gattorna and Day 1993), while a lean delivery emphasises a cost-effective and on-time handover with no delay or quality issues. Measurement of logistics is an important step to performance improvement. Performance measurement is a process of quantifying the efficiency and effectiveness of past actions, while a performance measure is a parameter used to quantify the efficiency and/or effectiveness of past actions ((Neely, Adams et al. 2002). In the early 1990s, project success was considered to be linked to performance measures, which in turn were linked to project objectives. At the project level, success was measured by the project duration, monetary cost and project performance, the so called project management "iron triangle" (Atkinson 1999). In addition to these basic criteria, researchers advocated that measures for construction project success should also include project psychosocial outcomes (such as participants' satisfaction level, and safety), timedependent dimensions, and perspectives of stakeholders (i.e. owner, developer, contractor, user and the general public) (Shenhar, Levy et al. 1997, Lim and Mohamed 1999).

Previous studies indicate that performance measurement has a notable effect on the development and effectiveness of benchmarking (Costa, Formoso et al. 2006). This is mainly because tracking performance identifies uncompetitive management practices which promotes investigation of changes. Sillanpää (2015) claimed that measuring the supply chain is the basis of developing it. KPIs are general indicators of performance that focus on critical aspects of outputs or outcomes. For performance measurement to be effective, the measures or indictors must be accepted, understood and owned across the organisation (Collin 2002). Also, only a limited and manageable number of KPIs is maintainable for regular use. Having too many and/or too complex KPIs can be time and resource consuming, data collection thus has to be as simple as possible. Another essential criterion of a KPI is that it can translate practices and measures into practical knowledge and make it possible to identify and adopt superior performance standards (Costa, Formoso et al. 2006). 
Wegelius-Lehtonen (2001) suggests a framework for performance measurement in construction logistics, that categorised the eight measurements (project time efficient, value added, subcontracting percentage, number of invoices per day, amount of small invoices, disposal costs, reply percentage of tenders, and amount of changes in subcontract) into two dimensions: use of measure and focus of measure. Further development of this framework has not yet occurred, which suggested that the indicators were not effective enough to practice at construction sites. Indeed, the construction firms do not usually have continuous data collection systems for logistics measures (Vogl and Abdel-Wahab 2014). In New Zealand, the practice in industry is in line with this claim (Page and Norman 2014). Research points towards the fact that the construction industry does not effectively address, or have the skills to solve, logistics problems. At present, the lack of knowledge is masked by a lack of immediacy in recognising that there is a problem at all. It is recognised that major barriers about awareness of logistics costs include invisible logistics costs, disconnect between investment in construction logistics and benefit, and no recorded data relating to logistics performance (Blumenthal and Young 2007, Omar, Hassan et al. 2009).

\section{RESEARCH METHODOLOGY}

The guiding purpose of this study was to contribute to the knowledge about measuring logistics performance by setting a KPI using the number of vehicle movements to the construction site. The focus of the work was on identification of the main performance aspects measured by the total numbers of vehicle movements. It also sought to understand why vehicle movements can be used as a KPI, as well as questions of how to use the vehicle movements in improving logistics performance. A case study with a qualitative and quantitative approach was used as this approach is appropriate when the research problem requires understanding of complex phenomena that are not controlled by the researcher and when the research questions have a how and why nature (Yin 2009).

It is important to select a critical case that can explicitly demonstrate. The choice of a commercial project in the largest city (by population and area) in NZ reflects typical problematic issues for construction logistics which critically demonstrates the "howproblem" (Yin 2009). The case study described in this paper was a commercial project hosted by a University located in central Auckland, implying special requirements in terms of logistics and physical distribution. Auckland is notable for its "Urban Sprawl" (Ministry of the Environment 2005). The city also has a substantial reliance on road transportation since public transport system has historically not kept up with population growth needs. The $\$ 100$ million project consisted of a 13 level tower block with a rooftop plant room surrounded by a lecture theatre and student facility. The new construction integrated several existing buildings on campus. The construction had three stages: ground works, structure, and fit-out. The contract was fixed price, with the client being allowed certain flexibility in the scope without extra charge.

On-site observations were performed during construction, as well as during weekly coordination meetings held between the main contractor and its subcontractors. These were documented through notes, photographs and audio recording. The depicted scenes gave an opportunity for participants to reflect on specific situations in retrospect. As noted by Scott and Garner (2013), observing behaviour gave opportunities to make sense of a larger context and draw conclusions that the individual subjects might have had difficulty noticing. Extensive observations were also made on the construction site to confirm information retrieved in the literature; the on-site observations also enabled information gathering that were unable to gather otherwise. 
Special attention has been paid to the numbers and patterns of vehicle movements, since it was expected that appropriate interventions to improve construction logistics could be identified through analysing these elements. The vehicle movements were recorded by the gates-person on the site. Details such as delivery company name, date, time, truck type, materials, and activities were noted on printed tables. These details were then transferred to electronic documents and analysed using MS Excel and MS Access.

These data were analysed as a whole, reduced to focus on the main objective of the paper and then presented in a synthesised form.

\section{KEY FINDINGS}

For the period of construction, the total number of vehicle movements to the observed site was approximately 6,300. These vehicles were from 257 difference firms. Vehicle movements were recorded in total for 334 days over 58 weeks. The different project stages and particular construction processes had profound signatures in the types, numbers and frequency of delivery vehicles entering the site.

Vehicles arrived at the site were classified in four categories, small (short and light two-axles vehicle, such as car, van and utilities), medium (short towing three to five axles vehicle, such as car with trailer), large (medium and heavy vehicle, 5.5 to $14.5 \mathrm{~m}$ with two to four axles), and very large (long and heavy vehicles, 11.5 to $19.0 \mathrm{~m}$ with three to six axles) (AUSTROADS 2006).

Similarly, time of delivery was analysed using same batch of data. The deliveries occurring throughout the day following no specific pattern. The histogram appears to be multimodal and skewed normal. The histogram also shows that almost one fifth of arrivals occur before 8:00 am while $55.8 \%$ of the arrivals occur during either in the early morning (08:00am to 10:00am) or early afternoon (12:00am to 2:00 pm). Indeed, vehicles arrivals on delivery points start after 6 and increase rapidly before peaking at the time interval between 9am to 10am. Then, taper down as time passes creating a strongly skewed distribution. In the studied project, $67.8 \%$ of delivers took place before midday.

The deliveries were most carried out from $8 \mathrm{am}$ to 11am (38.2\%), which overlaps with the peak time of city traffic from $7 \mathrm{am}$ to $9 \mathrm{am}$. These truck movements not only put extra burden on the existing saturated city traffic, but also reduce logistics efficiency. Some truck drivers complained about the tight space for manoeuvring in the city roads during peak traffic. These construction vehicle movements impose negative economic, social and environmental impacts by adding to the problem of congestion and environmental pollution.

Vehicle movements of each subcontractor were then further analysed to measure various aspects of logistics performance. In this paper, the data of ready-mix-concrete (Supplier A) are used to demonstrate that vehicle movement can be utilised to measure transportation costs. Figure 1 illustrates the delivery time carried out by Supplier A. The deliveries were mostly carried out during $2 \mathrm{pm}$ to $3 \mathrm{pm}$ (135 of 988 deliveries), and 9am to 11am (127 and 116 deliveries respectively). The diagram indicates that supplier A attempted to avoid the peak times of city traffic. It was confirmed by site manager that Supplier A was very cautious about concrete pouring time. They coordinated with the main contractor before each pouring day and tried to avoid traffic peak hours delivery. It is not surprising since ready-mix-concrete is a time sensitive product. 


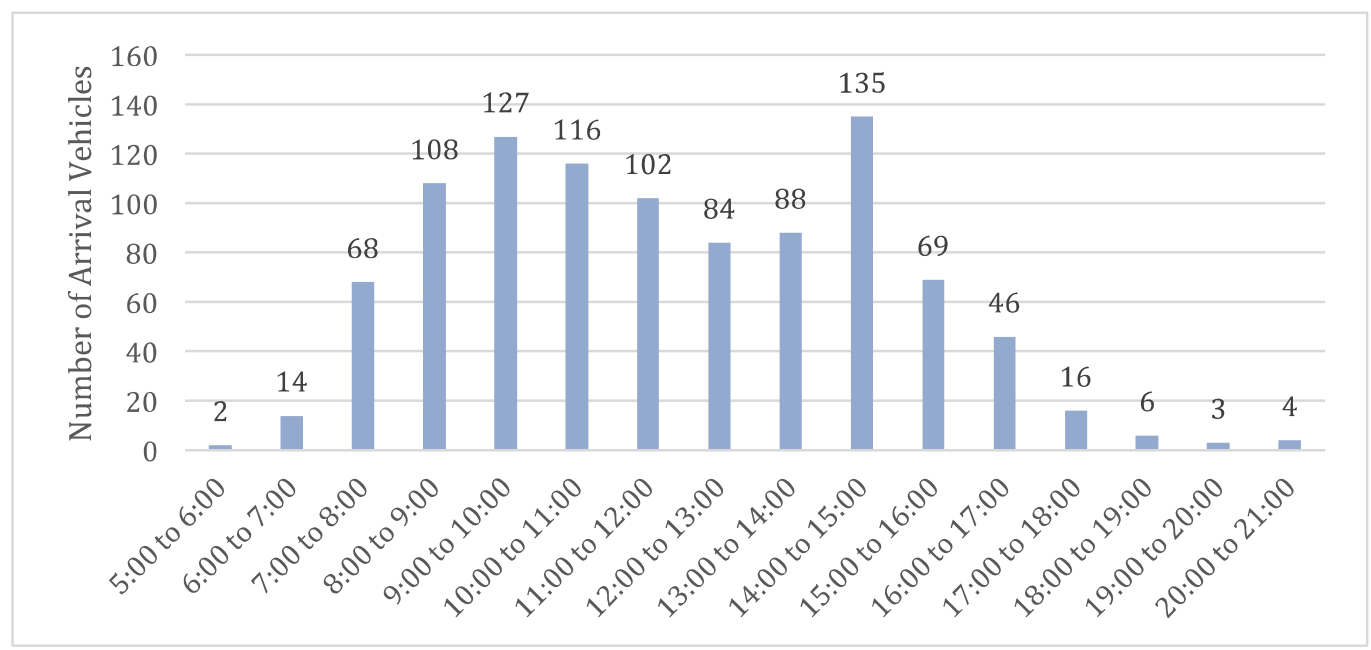

Figure 1: Vehicle arrival time -- Supplier A

$$
\text { Cost }_{\text {Transportation }}=\sum_{n=1}^{i} C O_{i} \times D_{i}
$$

Where: Cost $_{\text {Transportation }}$ is the total transportation costs, $\mathrm{CO}_{\mathrm{i}}$ is the vehicle operating cost of the $\mathrm{i}^{\text {th }}$ trip, $\mathrm{D}_{\mathrm{i}}$ is the distance between the site and the depot of the $\mathrm{i}^{\mathrm{th}}$ trip.

The vehicle operation costs vary with the size of the vehicle. For example, using the data for the ready-mix-concrete supplier, 952 large vehicles and 36 very small vehicles were made into the site. The concrete plant is located $4.1 \mathrm{~km}$ away from the construction site, giving $8.2 \mathrm{~km}$ for a round trip. There is no data for concrete mixer truck operating costs in New Zealand; the counterpart in the United States were adopted for this research (Zagula, Hinkle et al. 2012). The estimated operating cost for a concrete mixer is approximate $\$ 9.2 / \mathrm{km}$, which includes fuel, maintenance, accident repair, road call, drum chipping and driver's wages. Similarly, for a small vehicle, estimated operating cost is $\$ 1.2$ covering standing costs, fuel, tyres, service, and registration. Hence, the transportation costs for Supplier A for this project is $\$ 112,495$. The total amount of concrete supplied to the project is $4,865 \mathrm{~m} 3$, costing $\$ 1.05 \mathrm{~m}$. Accordingly, it is calculated that the transportation costs are $10.8 \%$ of the product selling price.

In the construction industry, the choice of material was primarily made on the basis of lowest per unit cost "as delivered". The cost of transportation was not isolated, and thus cannot be measured. Using data recorded at the site of vehicle movements, the "invisible" transportation costs for each firm and the whole project were able to be identified. The transportation costs lean to higher end of percentage of the production selling price comparing to previous research (4 to 10\%, according to Coyle, Bardi et al (2003)).

\section{DISCUSSION}

The main aim of this paper was to propose using vehicle movements as a KPI for monitoring and improving logistics performance, considering that KPIs are seen to be essential to measure and control construction performance.

Based on the empirical analyses and the generic body of knowledge concerning logistics and lean construction, the key findings indicate the construction logistics 
management practices in the studied project would be regard as "traditional" in that they are typically uncontrolled, disruptive and uncoordinated. The congested delivery time slot is evidence that there was not much communication or coordination regarding logistics among the subcontractors and material suppliers. These traditional practices are mainly caused by industry related issues. The construction industry is a project-oriented industry with many players. All major players, such as main contractors, subcontractors, and material suppliers, look at the logistics process from their own point of view. From the logistics process aspect, the studied project can be seen as independent project stages with different actors without a common goal. Thus, implementing performance measurement provides an opportunity for benchmarking and performance control.

Furthermore, the data analyses provide insights on the underlying drivers of performance. This is achieved by tangible and intangible inputs to leading performance measures that can be used to predict logistics performance, which may offer industry guidance on how to improve performance. Equipped with the information, such as peak delivery time, firms can evaluate the busiest delivery hours at the site and schedule deliveries accordingly to avoid long waiting times. The findings also give a snap shot of the interface between site activities and various material and plant supply chains, which is essential in lean construction (Vrijhoef and Koskela 2000).

Data analysis in this project demonstrates that the proposed indicator is simple and straightforward to establish a project logistics performance measurement system and incorporate the measures into the project routine. It is evident that measures can be translated into practical knowledge effectually by using a simple spreadsheet. Since the indicator focuses on data at the operational level, the interpretation of the data is straightforward and highly applicable to site management. Also, the proposed measurement is relevant to management in their daily work and is a means for continuous performance improvement on projects. The translation from practices and measures into practical knowledge makes it possible for the management team to identify and adopt a best performance standard.

\section{CONCLUSIONS}

This paper has discussed initiatives to develop a performance measurement indicator for benchmarking logistics performance in construction projects. Vehicles delivering materials and removing waste at the construction site physically link the supply logistics and site logistics. Vehicle movements thus could reflect the potential issues in both types of logistics. Using the numbers of vehicle movements as a KPI, the evidence provided in the case study demonstrates the indictor can effectively and efficiently describe and monitor site logistics performance. This study has pointed out benefits and opportunities for taking initiatives and implementing such a KPI.

The work presented here assess the ability of vehicle movement as a KPI in measuring the "invisible" transportation costs. In this project, the transportation costs of ready-mixconcrete, which is JIT delivery, is more than $10 \%$ of the material selling price. It is reasonable to assume that the project transportation costs would be much higher than this number, since the majority of the contractors delivered materials ad hoc. Based on the implications drawn, the findings of this study have the potential to be implemented by construction management companies building recorded database and thus to increase the likelihood of logistics management adoption by the construction industry.

It is arguable that logistics is far more than transportation costs. Thus, the major limitation using vehicle number as KPI is its ability of measuring restricted aspects of 
construction logistics. However, the research suggests the proposed indicator is simple and well designed to establish a project logistics performance measurement system and incorporate the measures into the project routine. It could be used as a starting point to measure the "invisible", bring the awareness to the industry and promote performance benchmarking at the operational level.

Moreover, the data presented in this paper focusing on one construction project. More inquiries are required in the form of case studies, such as other projects located in various geographic context, to generalise the material delivery pattern. Future studies also need to investigate whether firms with different supply chains characteristics demonstrate similar transportation costs and logistics efficiency

\section{REFERENCES}

Atkinson, R. (1999). "Project management: cost, time and quality, two best guesses and a phenomenon, its time to accept other success criteria." International Journal of Project Management 17(6): 337-342.

AUSTROADS (2006). Automatic vehicle classification by vehicle lengthe. Sydney, NSW, AUSTROADS.

Blumenthal, A. and A. Young (2007). Efficient construction logistics. Banbury, Oxon, Waste \& Resources Action Programme.

Collin, J. (2002). Measuring the success of building projects -- improved project delivery initiatives, Report for the Queensland Department of Public Works.

Constructing Excellence (NZ) Ltd (2008). New Zealand Construction Industry vision 2025: Initial research report. Wellington.

Costa, D. B., C. T. Formoso, M. Kagioglou, L. F. Alarcon and C. H. Caldas (2006). "Benchmarking initiatives in the construction industry: Lessons learned and improvement opportunities." Journal of Management in Engineering 22(4): 158-167.

Coyle, J. J., E. J. Bardi and C. J. L. Jr. (2003). The Management of Business Logistics: A Supply Chain Perspective. Mason, Ohio, Thomson Learning.

Coyle, J. J., E. J. Bardi and C. J. Langley (2003). Management of Business Logistics: A Supply Chain Perspective. Minneapolis, West Publishing Co.

Fellows, R., D. Langford, R. Newcombe and S. Urry (2002). Construction Management in Practice. London, Construction Press.

Gattorna, J. and A. Day (1993). "Strategic issues in logistics." International Journal of Physical Distribution \& Logistics Management 16(2): 3-42.

Jorgensen, B. and S. Emmitt (2009). "Investigating the integration of design and construction from a lean perspective." Construction Innovation 9(2): 225-240.

Lim, C. S. and M. Z. Mohamed (1999). "Criteria of project success: an exploratory reexamination." International Journal of Project Management 17(4): 243-248.

Ministry of the Environment (2005). New Zealand Urban Design Protocol. Wellington.

Neely, A., C. Adams and M. Kenerley (2002). The performance prism: the scorecard for measuring and management business success. London, Prentice-Hall.

OGC (2000). Achieving sustainability in construction procurement, The Sustainability Action Group of the Government Construction Clients' Panel (GCCP).

Omar, B., S. A. Hassan and T. Ballal (2009). Exploring context-awareness in the construction logistics services delivery. CIB W0728 26TH International conference, Istanbul. 
Key Performance Indicator for Managing Construction Logistics Performance

Page, I. and D. Norman (2014). Potential measures of productivity and performance at the firm, grouped firm and regional level. Wellington, BRANZ

Ministry of Business, Innovation \& Employment.

Scott, G. M. and R. Garner (2013). Doing qualitative research: designs, methods, and techniques. Boston, Pearson Education.

Shenhar, A. J., O. Levy and D. Dvir (1997). "Mapping the dimensions of project success." Project management journal 28(2): 5-13.

Sillanpaa, I. (2015). "Emprical study of measuring supply chain performance." Benchmarking: An International Journal 22(2): 290-308.

The KPI Working Group (2000). KPI report for the Minster for Construction. London, Department of the Environment, Transport and the Regions.

Vogl, B. and M. Abdel-Wahab (2014). "Measuring the construction industry's productivity performance: Critique of international productivity comparisons at industry level." Journal of Construction Engineering and Management November.

Vrijhoef, R. and L. Koskela (2000). "The four roles of supply chain management in construction." European Journal of Purchasing \& Supply Management 6(3-4): 169-178.

Wegelius-Lehtonen, T. (2001). "Performance measurement in construction logistics." International Journal of Production Economics 69(1): 107-116.

Yin, R. K. (2009). Case study research: Design and methods. Thousand Oaks, CA., Sage. Zagula, M., J. Hinkle, B. Mobley, D. Williams and G. M. Mullings (2012). 2012 National Ready Mixed concrete Association Fleet Benchmarking and Costs Survey, National Ready Mixed Concrete Association. 